Pure and Applied Mathematics Quarterly

Volume 5, Number 2

(Special Issue: In honor of

Friedrich Hirzebruch, Part 1 of 2)

$619-640,2009$

\title{
Involutions of Negatively Curved Groups with Wild Boundary Behavior
}

\author{
F. T. Farrell * and J.-F. Lafont ${ }^{\dagger}$ \\ Dedicated to Professor Friedrich Hirzebruch \\ on the occasion of his $80^{\text {th }}$ birthday.
}

\begin{abstract}
We are interested in examples of compact, locally CAT(-1) spaces $X$, and closed totally geodesic codimension two subspaces $Y$, with the property that $\partial^{\infty} \tilde{X}=S_{\infty}^{n+2}$, and $\partial^{\infty} \tilde{Y}=S_{\infty}^{n}$. We show that if the inclusion $S_{\infty}^{n} \hookrightarrow S_{\infty}^{n+2}$ induced by the inclusion $Y \subset X$ is knotted, then it is a totally wild knot (i.e. nowhere tame). We give examples where the inclusion $S_{\infty}^{n} \hookrightarrow S_{\infty}^{n+2}$ is indeed knotted. Furthermore, the examples $Y \subset X$ we construct can be realized as fixed point sets of involutive isometries of $X$, so that the corresponding totally wildly knotted $S_{\infty}^{n} \hookrightarrow S_{\infty}^{n+2}$ are fixed point sets of geometric involutions on $S_{\infty}^{n+2}=\partial^{\infty} \tilde{X}$. In the appendix, we also include a complete criterion for knottedness of tame codimension two spheres in high dimensional $(\geq 6)$ spheres.
\end{abstract}

Keywords: Hyperbolization, CAT(-1) space, boundary at infinity, involution, fixed set, knot.

Received January 3, 2006.

*This research was supported in part by the National Science Foundation.

${ }^{\dagger}$ This research was supported in part by the National Science Foundation, and in part by an Alfred P. Sloan research fellowship. 


\section{InTRODUCTION.}

For a space $Z$ that arises as the boundary at infinity of a $\delta$-hyperbolic group $\Gamma$, it is of some interest to determine which self-homeomorphisms of $Z$ are algebraic, i.e. are induced by an automorphism of $\Gamma$. Likewise, for a space $Z$ that arises as the boundary at infinity of the universal cover of a compact locally CAT(-1) space $X$, one can try to determine which self-homeomorphisms of $Z$ are geometric, i.e. are induced by isometric self-maps of $X$. Note that geometric selfhomeomorphisms induce algebraic self-homeomorphisms (by setting $\Gamma=\pi_{1}(X)$ ). In a previous paper [11], the authors constructed geometric involutions of spheres whose fixed point set had infinitely generated (Čech) cohomology. In this note, we analyze the geometry of fixed point sets of geometric involutions in the special case where $Z=S^{n+2}$ and the fixed point sets are embedded codimension two spheres.

Before stating our results, we remind the reader of a few basic facts about embeddings $S^{n} \hookrightarrow S^{n+2}$. For any such embedding, Alexander Duality tells us that the space $S^{n+2}-S^{n}$ is a homology $S^{1}$, hence there is a canonical infinite cyclic cover $S^{n+2}-S^{n} \rightarrow S^{n+2}-S^{n}$ arising from the abelianization $\pi_{1}\left(S^{n+2}-S^{n}\right) \rightarrow$ $H_{1}\left(S^{n+2}-S^{n}\right) \cong \mathbb{Z}$. An embedding $S^{n} \hookrightarrow S^{n+2}$ is unknotted if the complement $S^{n+2}-S^{n}$ is homeomorphic to $S^{1} \times \mathbb{R}^{n+1}$, otherwise it is said to be knotted. Note that, for an unknotted embedding, the infinite cyclic cover $S^{n+2}-S^{n}$ is homeomorphic to $\mathbb{R} \times \mathbb{R}^{n+1}$, while the fundamental group of the complement is $\pi_{1}\left(S^{n+2}-S^{n}\right)=\mathbb{Z}$. This gives sufficient conditions for an embedding $S^{n} \hookrightarrow S^{n+2}$ to be knotted, namely if either:

- $\pi_{1}\left(S^{n+2}-S^{n}\right) \neq \mathbb{Z}$,

- or $H_{s}\left(\widehat{S^{n+2}-} S^{n}\right) \neq 0$ for some $s \neq 0, n$,

then the embedding is knotted. Conversely, when $n \geq 4$ and the embedding is smooth, one can use work of Browder-Levine [4] to show that the above conditions are also necessary (see Theorem 4.1 in the Appendix for a proof).

An embedding $S^{n} \hookrightarrow S^{n+2}$ is said to be tame at a point $p \in S^{n}$ provided there exists a neighborhood $U$ of $p$ in $S^{n+2}$ with the property that $\left(U, S^{n} \cap U\right)$ is homeomorphic to $\left(\mathbb{R}^{n+2}, \mathbb{R}^{n}\right)$ (where the $\mathbb{R}^{n} \hookrightarrow \mathbb{R}^{n+2}$ is the embedding into the first factor of $\mathbb{R}^{n+2} \cong \mathbb{R}^{n} \times \mathbb{R}^{2}$ ). In other words, the embedding is "collarable" near the point $p$. A point $p \in S^{n}$ that is not tame is said to be wild. An 
embedding is tame provided it is tame at every point, and is totally wild if it is wild at every point. Examples of non-tame embeddings of 2-spheres in 3-spheres include the Alexander horned sphere, the Antoine wild sphere and the Fox-Artin ball (see Bing [3] for details). A result which will be frequently used is that codimension two tame embeddings have tubular neighborhoods; this is due to Kirby-Siebenmann [15] when the ambient dimension is $\neq 4$, and to FreedmanQuinn (Section 9.3 in [12]) in the remaining case. We are now ready to state our main results.

Theorem 1.1. Let $\Gamma$ be a $\delta$-hyperbolic group, $\Lambda \leq \Gamma$ a quasi-convex subgroup. Assume that $\partial^{\infty} \Gamma=S_{\infty}^{n+2}, \partial^{\infty} \Lambda=S_{\infty}^{n}$, where $n \neq 0,3$, and that the embedding $S_{\infty}^{n} \hookrightarrow S_{\infty}^{n+2}$ induced by the inclusion $\Lambda \leq \Gamma$ is knotted. Then the embedding is a totally wild knot.

This allows us to answer a question we asked in our previous paper [11]:

Corollary 1.1 (Generalized Smith Conjecture for algebraic involutions). Let $\tau: S^{n+2} \rightarrow S^{n+2}$ be an algebraic self-homeomorphism $(n \neq 0,3)$ of finite order, and assume that the fixed point set is an embedded $S^{n} \hookrightarrow S^{n+2}$. If the embedding is tame, then $S^{n} \hookrightarrow S^{n+2}$ is unknotted.

In the previous corollary, by an algebraic self-homeomorphism of finite order, we mean an algebraic self-homeomorphism induced by an automorphism of finite order. Theorem 1.1 also yields the immediate corollary:

Corollary 1.2. Let $X$ be a compact, locally $C A T(-1)$ space, $Y \subset X$ a totally geodesic subspace, $\tilde{Y}$ and $\tilde{X}$ their respective universal covers. Assume that $\partial^{\infty} \tilde{Y}=S_{\infty}^{n}, \partial^{\infty} \tilde{X}=S_{\infty}^{n+2}$, where $n \neq 0,3$, and that the embedding $S_{\infty}^{n} \hookrightarrow S_{\infty}^{n+2}$ induced by a lift $\tilde{Y} \subset \tilde{X}$ is knotted. Then the embedding is a totally wild knot.

Even in the special case of fundamental groups of negatively curved Riemannian manifolds, this gives the interesting:

Corollary 1.3. Let $M^{n}(n \geq 4, n \neq 6)$ be a closed, negatively curved Riemannian manifold, and let $\alpha \in \operatorname{Aut}\left(\pi_{1}\left(M^{n}\right)\right)$ have finite order. Denote by $\alpha_{\infty}: S_{\infty}^{n-1} \rightarrow$ $S_{\infty}^{n-1}$ the induced involution on the sphere at infinity of the universal cover $\tilde{M}^{n}$. Then the fixed point set of $\alpha_{\infty}$ cannot be a tame codimension two knot. 
The next reasonable question is whether one can find examples satisfying the hypotheses of Theorem 1.1. This is addressed in the following:

Theorem 1.2. Let $S^{n} \hookrightarrow S^{n+2}$ be a tamely knotted codimension two PL-embedding, with $n \geq 5$. Let $X$ be a strict hyperbolization of the suspension of $S^{n+2}$, and $Y \subset X$ a corresponding strict hyperbolization of the suspension of $S^{n}$. Then the embedding $\partial^{\infty} \tilde{Y} \subset \partial^{\infty} \tilde{X}$ is a knotted $S_{\infty}^{n}$ in $S_{\infty}^{n+2}$. By the previous theorem, this knot must be a totally wild knot.

From our construction in the previous Theorem, we can also exhibit these knots as fixed point sets of geometric actions:

Corollary 1.4. Let $\tau$ be a PL involution of a sphere $S^{n+2}$ whose fixed point set is a tamely knotted codimension two PL-embedded sphere $S^{n}(n \neq 3)$. Let $X$ be the strict hyperbolization of the suspension of $S^{n+2}, \tau_{h}$ the induced involution on $X$, and $\tilde{\tau}_{h}$ a lift of the involution to an involution of the universal cover $\tilde{X}$. Finally, let $\tau_{\infty}$ be the induced involution on the boundary at infinity of the universal cover $\tilde{X}$. Then the fixed subset of $\tau_{\infty}$ is a knotted, totally wild embedding of $S^{n}$ in $S^{n+2}$.

A consequence of the previous corollary is the following:

Corollary 1.5. There exists a smooth closed manifold $M^{n+3}(n \geq 5)$, supporting a locally $C A T(-1)$ metric, and having an involution $\sigma \in P L\left(M^{n+3}\right)$, with the property that $\sigma$ is not homotopic to an involutive isometry for any negatively curved Riemannian metric on $M^{n+3}$.

It is conceivable that the smooth manifold $M^{n+3}$ in the previous corollary might not even support any Riemannian metric of negative curvature (see Section 5). Before starting with the proofs, we make a few remarks about our results.

Remark 1. Note that geometric involutions induce algebraic involutions, by setting $\Gamma=\pi_{1}(X)$. As such, our Corollary 1.1 also applies to geometric involutions, and yields that for geometric involutions whose fixed point sets are $S^{n} \hookrightarrow S^{n+2}$, tameness implies the embedding is unknotted. Conversely, the examples we give in Corollary 1.3 of knotted fixed point sets for geometric involutions automatically give us knotted fixed point sets for algebraic involutions. 
Remark 2. Recall that, in high dimension, examples of PL involutions on spheres with tamely knotted codimension two spheres as fixed point sets exist, by results of Giffen [13]. In particular, there are examples satisfying the hypotheses of our Corollary 1.4. Concrete examples can be obtained by looking at Brieskorn spheres. Hirzebruch constructed the first such examples in [14]; later Lü expanded on this construction in [17]. This should be contrasted with the situation in $S^{3}$, where by the celebrated solution to the Smith conjecture (see Bass-Morgan [1]), a tamely knotted $S^{1}$ cannot be the fixed point set of a smooth $\mathbb{Z}_{p^{-}}$action on $S^{3}$. In contrast, our Corollary 1.1 shows that tamely knotted codimension two spheres cannot arise as fixed point sets of algebraic actions.

Remark 3. Our approach to Theorem 1.2 is based on the general philosophy that properties of the links in a simplicial complex $X$ give corresponding local properties for the boundary at infinity $Z$ of the universal cover of the hyperbolization of the simplicial complex. Various other results that are based on the same philosophy include:

- if the links of $X$ are spheres, then the boundary $Z$ is a Čech cohomology sphere (Bestvina [2]).

- if the links of $X$ are PL-spheres, then the boundary $Z$ is a topological sphere (Davis \& Januszkiewicz [7]).

- bad homology in the links can propagate to give infinitely generated Čech cohomology in the boundary (see Farrell-Lafont [11] for a more precise statement).

It would be interesting to construct other types of pathologies via the hyperbolization technique. It would also be interesting to see if other methods could be used to construct similar pathologies.

Remark 4 . This paper was motivated in part by the following more specific question (which is still open). Let $\Gamma=\pi_{1}(M)$ where $M$ is a closed negatively curved Riemannian manifold, and let $\alpha: \Gamma \rightarrow \Gamma$ be an automorphism with $\alpha^{2}=I d_{\Gamma}$. Is $\alpha$ induced by an involution of $M$ ? That is to say, does there exist a selfhomeomorphism $f: M \rightarrow M$ with $f^{2}=I d_{M}$, and $f_{\sharp}=\alpha$ ?

\section{Non-existence of tame KnOts.}

We start by giving a proof of Theorem 1.1: 
Proof (Theorem 1.1). We have $\Gamma$ a $\delta$-hyperbolic group, and $\Lambda \leq \Gamma$ a quasi-convex subgroup. We are assuming that $\partial^{\infty} \Gamma=S_{\infty}^{n+2}, \partial^{\infty} \Lambda=S_{\infty}^{n}$, and since $\Lambda$ is a quasi-convex subgroup of $\Gamma$, we get an embedding $S_{\infty}^{n} \hookrightarrow S_{\infty}^{n+2}$. We start by showing that, if the embedding is tame, then the embedding is the trivial knot. To argue by contradiction, we make the:

Assumption: $S_{\infty}^{n}$ is tame in $S_{\infty}^{n+2}$.

Since the embedding is tame, we let $N$ be a closed tubular neighborhood of $S_{\infty}^{n}$ in $S_{\infty}^{n+2}\left(N_{\circ}\right.$ its interior), and let $W=S_{\infty}^{n+2}-N$. Note that $W^{n+2}$ is an open manifold, homeomorphic to $S_{\infty}^{n+2}-S_{\infty}^{n}$, and the inclusion $W^{n+2} \subset S_{\infty}^{n+2}-S_{\infty}^{n}$ is a homotopy equivalence. For a subset $A \subset S_{\infty}^{n+2}-S_{\infty}^{n}$, we denote by $\widehat{A}$ the pre-image $p^{-1}(A)$ where $p: S_{\infty}^{n+2}-S_{\infty}^{n} \rightarrow S_{\infty}^{n+2}-S_{\infty}^{n}$ is the infinite cyclic cover defined in the introduction. Since the embedding was assumed to be a non-trivial tame knot, $n \neq 0,3$ and Theorem 4.1 in the Appendix (together with Remark 6 on pg. 19) tells us that either:

- $\pi_{1}(W) \neq \mathbb{Z}$, or

- $H_{s}(\widehat{W}) \neq 0$ for some $0<s, s \neq n$.

Let us consider the case where $\pi_{1}(W) \neq \mathbb{Z}$. Since the abelianization of $\pi_{1}(W)$ is $\mathbb{Z}$, we know that the group $\pi_{1}(W)$ is non-abelian. Note that $\Lambda$ acts on $\left(S_{\infty}^{n+2}, S_{\infty}^{n}\right)$ by homeomorphisms, and the dynamics of the action are sink/source (with the sinks and sources lying on $\left.S_{\infty}^{n}\right)$. Pick $g$ an element of infinite order in $\Lambda$, and $n$ sufficiently large so that we have $g^{n}(W) \subset N_{\circ}-S_{\infty}^{n}$. Since $g$ is a homeomorphism, we have that $g^{n}(W) \subset S_{\infty}^{n+2}-S_{\infty}^{n}$ is also a homotopy equivalence. But the inclusion factors through the inclusion $N_{\circ}-S_{\infty}^{n} \subset S_{\infty}^{n+2}-S_{\infty}^{n}$. Since $N$ is a tubular neighborhood of $S_{\infty}^{n}$, we see that $\pi_{1}\left(N_{\circ}-S_{\infty}^{n}\right)=\pi_{1}\left(S^{n} \times S^{1}\right)=\mathbb{Z}$ (if $n>1$ ) or $\mathbb{Z} \oplus \mathbb{Z}$ (if $n=1$ ), hence is always abelian. This yields a commutative diagram:

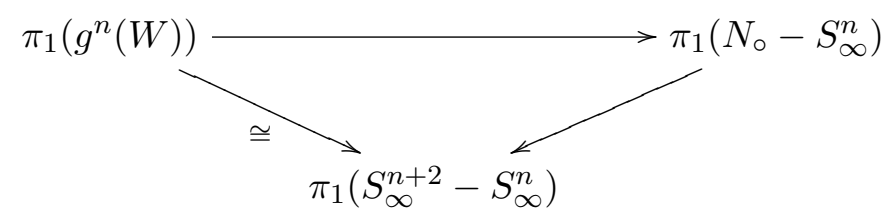


where the arrows are induced by the inclusions. Since $\pi_{1}\left(S_{\infty}^{n+2}-S_{\infty}^{n}\right)$ is nonabelian, but the isomorphism factors through an abelian group, we get a contradiction.

Next we consider the case where $H_{s}(\widehat{W}) \neq 0$ for some $0<s, s \neq n$. Arguing as before, we take $g$ to be an element of infinite order in $\Lambda$, and $n$ sufficiently large so that we have $g^{n}(W) \subset N_{\circ}-S_{\infty}^{n}$. Since $g$ is a homeomorphism, it preserves $H_{1}\left(S_{\infty}^{n+2}-S_{\infty}^{n}\right)$, and lifts to a self-homeomorphism of $S_{\infty}^{n+2}-S_{\infty}^{n}$ (which we also denote by $g$ ). Note that we have $\widehat{g^{n}(W)}=g^{n}(\widehat{W})$. Furthermore, we have that $\widehat{N_{\circ}-S_{\infty}^{n}}$ is homeomorphic to $S^{n} \times \mathbb{R}^{2}$, forcing $H_{s}\left(\widehat{N_{\circ}-S_{\infty}^{n}}\right)=0$ (since $s>0, s \neq n)$. We now have a commutative diagram:

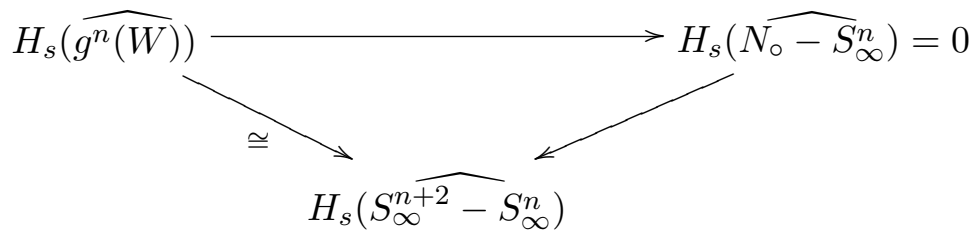

where the arrows are induced by the corresponding inclusions. Since $H_{s}(\widehat{W})$ was assumed to be non-zero, we again get a contradiction.

So in all cases, we see that the embedding $S_{\infty}^{n} \hookrightarrow S_{\infty}^{n+2}$ cannot be tame. Now let $p \in S_{\infty}^{n}$ be a wild point. Since the orbit of $p$ under the action of $\Lambda$ on the pair $\left(S_{\infty}^{n+2}, S_{\infty}^{n}\right)$ is dense in $S_{\infty}^{n}$, we immediately obtain that $S_{\infty}^{n}$ cannot contain any tame points, i.e. the embedding $S_{\infty}^{n} \hookrightarrow S_{\infty}^{n+2}$ is totally wild. This completes the proof of Theorem 1.1.

Proof (Corollary 1.1). Let $\tau$ be an automorphism of finite order of the $\delta$-hyperbolic group $\Gamma$, and $\tau_{\infty}$ the induced homeomorphism on $\partial^{\infty} \Gamma=S_{\infty}^{n+2}$. Assume that the fixed point set of $\tau_{\infty}$ is a tamely embedded codimension two sphere $S_{\infty}^{n} \hookrightarrow S_{\infty}^{n+2}$. We need to show it is unknotted.

First note that Proposition 2.1 in [11] shows that the fixed set of $\tau_{\infty}$ coincides with $\partial^{\infty}\left(\Gamma^{\tau}\right)$. A result of Neumann [18] ensures that $\Gamma^{\tau}$ is a quasi-convex subgroup of $\Gamma$. Applying Theorem 1.1, we see that this forces the fixed point set to be a trivial knot.

Proof (Corollary 1.2). This is immediate, since the hypotheses on $X, Y$ ensure that $\Gamma:=\pi_{1}(X), \Lambda:=\pi_{1}(Y)$ are $\delta$-hyperbolic groups, and that the embedding 
$\Lambda \leq \Gamma$ is quasi-convex. Since the embedding $\tilde{Y} \subset \tilde{X}$ is coarsely equivalent to the embedding $\Lambda \leq \Gamma$, we have a natural homeomorphism allowing us to identify $\left(\partial^{\infty} \tilde{X}, \partial^{\infty} \tilde{Y}\right)$ with the pair $\left(\partial^{\infty} \Gamma, \partial^{\infty} \Lambda\right)$. The corollary now follows from Theorem 1.1

Proof (Corollary 1.3). This follows immediately from Corollary 1.2.

\section{Existence of Wild KNOts.}

In this section, we focus on proving Theorem 1.2. A proof of Corollaries 1.4 and 1.5 will be given at the end of the section.

We start by observing that the links of vertices in both $\Sigma S^{n}$ and the corresponding $\Sigma S^{n+2}$ are PL-spheres. Since hyperbolization preserves the local structure of the links, we have that the links of vertices in both $X^{n+3}$ and $Y^{n+1}$ are PL-homeomorphic to spheres of dimension $S^{n+2}$ and $S^{n}$ respectively. By a result of Davis-Januszkiewicz [7], this implies that the boundaries at infinity of the universal covers $\tilde{X}^{n+3}$ and $\tilde{Y}^{n+1}$ are homeomorphic to $S^{n+2}$ and $S^{n}$ respectively. We want to show that the inclusion is a wildly embedded codimension two sphere. We use the subscript " $\infty$ " to distinguish the spheres at infinity from any of the other spheres that will appear in our proof. We will use $\bar{X}, \bar{Y}$ respectively to denote the compactified spaces $\bar{X}=\tilde{X} \cup S_{\infty}^{n+2}$ and $\bar{Y}=\tilde{Y} \cup S_{\infty}^{n}$.

Let $* \in \tilde{Y}$ be a lift of the hyperbolization of the (northern) suspension point, and let $S_{*}^{n}, S_{*}^{n+2}$ denote the links of the point $*$ in $\tilde{Y}, \tilde{X}$ respectively. As in the previous section, if $W$ is any subspace of $S_{\infty}^{n+2}-S_{\infty}^{n}$ (or of $S_{*}^{n+2}-S_{*}^{n}$ ), we denote by $\widehat{W}$ its inverse image in the appropriate infinite cyclic cover. Note that the restriction $\widehat{W} \rightarrow W$ is also an infinite cyclic covering projection.

Before starting with the argument, we introduce a little more notation: let $K_{*}$ denote an open metric ball of radius $\epsilon$ centered at $*$ in $\tilde{X}$, with $\epsilon$ chosen small enough so that $\partial K_{*}$ is the link $S_{*}^{n+2}$, and let:

$$
\rho: \bar{X}-K_{*} \rightarrow \partial K_{*}=S_{*}^{n+2}
$$

be the geodesic retraction.

The next proposition will be needed in order for us to apply Lefschetz duality to sets of the form $\rho^{-1}(U)$, and will primarily be used in the proof of Proposition 2 . 
Proposition 1. For each open set $U \subset S_{*}^{n+2}$, the map $\rho: \rho^{-1}(U) \rightarrow U$ is a proper homotopy equivalence.

Proof (Proposition 1). By a result of Edwards [9], it is sufficient to show that the pre-image $\bar{P}:=\rho^{-1}(p)$ is contractible for any point $p \in S_{*}^{n+2}$. Note that contractibility of the set $\bar{P}$ would be immediate if we knew that $P:=\bar{P} \cap X$ is a totally geodesic subspace of $X$. Indeed, if this was the case, the fact that the embedding is totally geodesic would imply that $\bar{P}$ is homeomorphic to the compactification $P \cup \partial^{\infty} P$ of $P$ viewed as a $\operatorname{CAT}(-1)$ space. But the latter is automatically contractible.

Unfortunately, $P$ is not quite totally geodesic. However, it is a contractible quasi-convex subset of $X$, in the sense that there exists a constant $C$ such that any geodesic segment with endpoints in $P$ lies in a $C$-neighborhood of $P$. To see this, we merely observe that the $\mathrm{CAT}(-1)$ space $X$ is automatically $\delta$-hyperbolic. Now given any two points $x, y \in P$, we consider the geodesic triangle with vertices $p, x, y$. Note that the geodesics $\overline{x p}$ and $\overline{y p}$ both lie in the set $P$, so that $\delta$ hyperbolicity tells us that the geodesic $\overline{x y}$ lies in a $C$-neighborhood of $\overline{x p} \cup \overline{y p} \subset P$ for some uniform constant $C$.

Now a quasi-convex subset of a $\delta$-hyperbolic space is still a $\delta$-hyperbolic space. Furthermore, the embedding of the quasi-convex subset extends to the boundary at infinity. This implies that $\bar{P}$ coincides with $P \cup \partial^{\infty} P$ (where we view $P$ as a $\delta$-hyperbolic space). We can now homotope $P \cup \partial^{\infty} P$ into the set $P$ (since $\partial^{\infty} P$ is a Z-set). Finally, applying the geodesic retraction to $P$, we obtain that the set $\bar{P}$ is indeed contractible, concluding the proof of Proposition 1.

To simplify notation, for a set $Z \subset S_{*}^{n+2}$, we use $Z_{\infty}$ to denote $\rho^{-1}(Z) \cap S_{\infty}^{n+2}$, i.e. $Z_{\infty}$ is the subset of the sphere at infinity that projects to $Z$ under geodesic retraction. Our next proposition allows us to relate the topology of an open set $U$ in $S_{*}^{n+2}-S_{*}^{n}$ with the topology of the set $U_{\infty}$.

Proposition 2. For each open set $U \subset S_{*}^{n+2}-S_{*}^{n}$, the map $\rho_{\infty}: U_{\infty} \rightarrow U$ is a proper homotopy equivalence (where $\rho_{\infty}$ is the restriction of the map $\rho$ to the set $\left.U_{\infty}\right)$.

Proof (Proposition 2). We note that $U_{\infty}$ is the inverse limit of the sequence $\left\{U_{r}\right\}$, where the $U_{r}$ are the intersections of $\rho^{-1}(U)$ with spheres of radius $r$ centered at the point $*$ (in particular, $U_{\epsilon}=U$ ), and the bonding maps are the restriction 
of the geodesic retraction $\rho_{r, s}: U_{r} \rightarrow U_{s}$ (with $\epsilon \leq s \leq r<\infty$ ). We denote by $\rho_{\infty, j}: \lim _{\longleftarrow}\left\{U_{i}\right\} \rightarrow U_{j}$ the canonical map from the inverse limit to the individual $U_{j}$ (and observe that $\rho_{\infty, \epsilon}$ coincides with the map $\rho_{\infty}$ ).

We say a map is cell-like provided the pre-image of each point has the same shape as a point (where shape refers to the functor from the homotopy category to the shape category). Davis-Januszkiewicz have shown that the bonding maps $\rho_{r, s}$ are cell-like maps (see Section 3 in [7]). A basic property of the shape functor is that it commutes with inverse limits (see Dydak-Segal [8]). So under our hypotheses, we see that:

$$
\begin{aligned}
& \operatorname{Shape}\left(\rho_{\infty, j}^{-1}(x)\right)=\operatorname{Shape}\left(\lim _{\longleftarrow}\left\{\rho_{i, j}^{-1}(x)\right\}\right)=\lim _{\longleftarrow}\left\{\operatorname{Shape}\left(\rho_{i, j}^{-1}(x)\right)\right\} \\
& =\lim _{\longleftarrow}\{\operatorname{Shape}(x)\}=\operatorname{Shape}(x)
\end{aligned}
$$

where $x \in U$ is arbitrary. This implies that the canonical map $\rho_{\infty, \epsilon}=\rho_{\infty}$ from $\lim _{\longleftarrow}\left\{U_{i}\right\}=U_{\infty}$ to $U$ is cell-like. Now a result of Edwards (Section 4 in [9]) asserts that a cell-like proper surjection of ANR's is a proper homotopy equivalence, concluding the proof of Proposition 2.

From the previous two propositions, we can immediately obtain:

Proposition 3. For each open set $U \subset S_{*}^{n+2}-S_{*}^{n}$, the inclusion map $i^{+}: U_{\infty} \hookrightarrow$ $\rho^{-1}(U)$ is a proper homotopy equivalence.

Proof (Proposition 3). We observe that we have a commutative diagram:

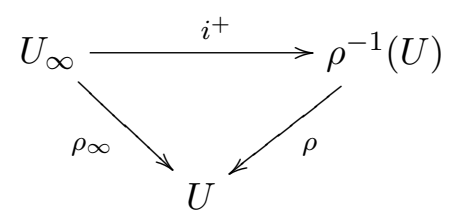

From Propositions 1 and 2, we know that both of the maps $\rho_{\infty}$ and $\rho$ are proper homotopy equivalences. This forces $i^{+}$to likewise be a proper homotopy equivalence.

Remark 5. We point out that an immediate consequence of Proposition 2 is that the map $\rho_{\infty}: U_{\infty} \rightarrow U$ is a near-homeomorphism (i.e. it can be approximated arbitrarily closely by homeomorphisms). This is a consequence of the fact that $\rho_{\infty}$ is cell-like, along with the result of Siebenmann [20] that cell-like maps between 
manifolds are near-homeomorphisms. However, this stronger result will not be used in the rest of this paper.

In the paper of Davis-Januszkiewicz [7], the Siebenmann result was used at the level of the maps $\rho_{r, s}$, giving that the boundary at infinity was the inverse limit of topological spheres with bonding maps that were near-homeomorphisms. A result of Brown [5] implies that the inverse limit of homeomorphic compact spaces, with bonding maps that are near-homeomorphisms, has to be homeomorphic to the spaces in question. We cannot apply Brown's result in our setting, as we are working with open sets $U$.

Finally, we point out that the argument in Proposition 2 can be used to show the following: if one has an inverse limit of homeomorphic manifolds, with bonding maps which are cell-like, then the inverse limit is homeomorphic to the manifold in question. In the situation where the manifolds are compact, this follows from Brown's result. In the non-compact case, we reach the same conclusion as Brown, but require the stronger hypothesis on the bonding maps.

Continuing our proof, notice that $\left(\rho^{-1}(U) ; U, U_{\infty}\right)$ is an open cobordism (of dimension $n+3$ ); i.e.

$$
\partial\left(\rho^{-1}(U)\right)=U \amalg U_{\infty} .
$$

We will use $i^{+}: U_{\infty} \hookrightarrow \rho^{-1}(U)$ and $i^{-}: U \hookrightarrow \rho^{-1}(U)$ to denote the respective inclusions. Note that, by Proposition 1 and Proposition 3, the maps $i^{ \pm}$are homotopy equivalences. Now if $U \subset S_{*}^{n+2}-S_{*}^{n}$, then the pullback of the covering space $\widehat{U} \rightarrow U$ via $\rho$ gives a canonical infinite cyclic cover $\widehat{\rho^{-1}(U)} \rightarrow \rho^{-1}(U)$. In fact, we have a commutative diagram:

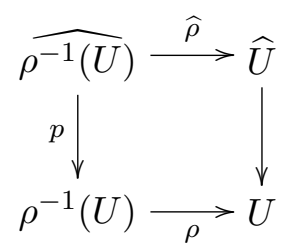

where $\widehat{\rho}$ is the lift of $\rho$. Note that $\widehat{\rho}$ is a proper homotopy equivalence which is also a retraction. Duality considerations yield the following important fact:

Proposition 4. $\widehat{U_{\infty}}=p^{-1}\left(U_{\infty}\right)$, where $p$ is the covering projection in the commutative diagram above. 
Proof (Proposition 4). Elementary covering space theory reduces the proof of Proposition 4 to showing that the inclusion map $\sigma^{+}: \rho^{-1}\left(S_{*}^{n+2}-S_{*}^{n}\right) \cap S_{\infty}^{n+2} \subset$ $S_{\infty}^{n+2}-S_{\infty}^{n}$ induces an isomorphism on $H_{1}(-; \mathbb{Z})$. For this, we need that $\bar{X}-K_{*}$ and $\bar{Y}-K_{*}$ are both manifolds, and if $n \geq 5$, then by the h-cobordism theorem they are automatically homeomorphic to $S^{n+2} \times[0,1]$ and $S^{n} \times[0,1]$ respectively. Set $W^{n+3}=\bar{X}-\left(K_{*} \cup \bar{Y}\right)$, and note that $W$ is an open cobordism with two boundary components: $\partial^{+} W=S_{\infty}^{n+2}-S_{\infty}^{n}$ and $\partial^{-} W=S_{*}^{n+2}-S_{*}^{n}$. We make the:

Claim: Both inclusion maps $\tau^{ \pm}: \partial^{ \pm} W \subset W$ induce isomorphisms on $H_{1}(-; \mathbb{Z})$.

Assuming the validity of this claim, we now proceed to complete the verification of Proposition 4. Let $\sigma: \rho^{-1}\left(\partial^{-} W\right) \subset W$ denote the inclusion map, and consider the following commutative diagram of inclusions:

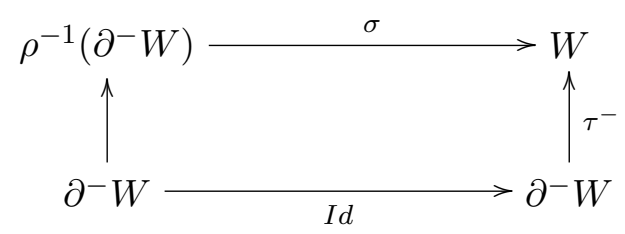

Hence Proposition 1 (with $U=\partial^{-} W$ ) and the Claim show that $\sigma_{*}$ is an isomorphism on $H_{1}(-; \mathbb{Z})$. Next consider the commutative diagram of inclusions:

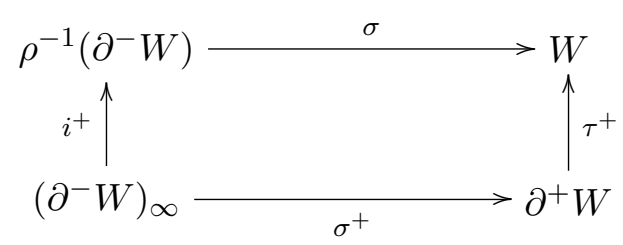

By the Claim, $\tau_{*}^{+}$is an isomorphism on $H_{1}$, and we've just shown that $\sigma_{*}$ is an isomorphism. By Proposition 3, we have that $i_{*}^{+}$is an isomorphism on $H_{1}$. This implies that $\sigma_{*}^{+}$is an isomorphism on $H_{1}$.

Hence to complete the proof of Proposition 4, it remains to establish the Claim. To do this, it is clearly enough to show that both $H_{i}\left(W, \partial^{ \pm} W\right)=0$, for all $i$. We will do this only for the case $H_{i}\left(W, \partial^{-} W\right)$ since the proof of the other case is 
completely analogous. By Lefschetz duality, we have:

$$
H_{i}\left(W, \partial^{-} W\right) \cong H_{c}^{(n+3)-i}\left(W, \partial^{+} W\right) .
$$

Hence it is equivalent to show that $H_{c}^{j}\left(W, \partial^{+} W\right)=0$ for all $j$. And since $H_{c}^{j}\left(W, \partial^{+} W\right)$ is isomorphic to $H_{c}^{j}\left(W-\partial^{+} W\right)$ (see below), it suffices to show that $H_{c}^{j}\left(W-\partial^{+} W\right)=0$ for all $j$.

Let $X_{*}$ and $Y_{*}$ denote $X-\bar{K}_{*}$ and $Y-\bar{K}_{*}$ respectively (note that $X_{*}$ and $Y_{*}$ are homeomorphic to $S^{n+2} \times[1, \infty)$ and $S^{n} \times[1, \infty)$ respectively). Now consider the exact sequence in cohomology with compact supports for the pair $\left(X_{*}, Y_{*}\right)$ :

$$
H_{c}^{j}\left(X_{*}\right) \leftarrow H_{c}^{j}\left(X_{*}, Y_{*}\right) \leftarrow H_{c}^{j-1}\left(Y_{*}\right) .
$$

Because of Lefschetz duality

$$
H_{c}^{j}\left(X_{*}\right)=H_{c}^{j}\left(S^{n+2} \times[1, \infty)\right) \cong H_{(n+3)-j}\left(S^{n+2} \times[1, \infty), S^{n+2} \times 1\right)=0 .
$$

Similarly $H_{c}^{j-1}\left(Y_{*}\right)=0$, and therefore $H_{c}^{j}\left(X_{*}, Y_{*}\right)=0$ for all $j$. Recall that if $A$ is a closed subspace of $B$ (both locally compact spaces), then

$$
H_{c}^{j}(B, A) \cong H_{c}^{j}(B-A)
$$

(note that this was used in the previous paragraph). Hence

$$
H_{c}^{j}\left(X_{*}-Y_{*}\right)=0
$$

for all $j$. Since $X_{*}-Y_{*}=W-\partial^{+} W$, we have completed the proof of Claim, and hence also of Proposition 4.

In other words, the infinite cyclic covering spaces of $U_{\infty}$ induced from $S_{\infty}^{n+2}-S_{\infty}^{n}$ and $S_{*}^{n+2}-S_{*}^{n}$ are consistent. An immediate consequence of this is that, for the infinite cyclic coverings, we have analogues of Propositions 1,2, and 3. Since we will need the analogue of Proposition 2, we explicitly state it below. For any open set $U \subset S_{*}^{n+2}-S_{*}^{n}$, associated to the proper h-cobordism $\left(\rho^{-1}(U) ; U, U_{\infty}\right)$, we have a canonical infinite cyclic covering by the induced proper h-cobordism $\left(\widehat{\rho^{-1}(U)} ; \widehat{U}, \widehat{U_{\infty}}\right)$.

Proposition $2^{\prime}$ : The map $\widehat{\rho}: \widehat{U_{\infty}} \rightarrow \widehat{U}$ is a proper homotopy equivalence.

Next we note that, since hyperbolization preserves links, we have by construction that $S_{*}^{n} \subset S_{*}^{n+2}$ is a tame knot. In particular, since $n \neq 3$, we have (see Theorem 4.1 in Appendix) either: 
- $\pi_{1}\left(S_{*}^{n+2}-S_{*}^{n}\right) \neq \mathbb{Z}$, or

- $H_{s}\left(\widehat{S_{*}^{n+2}-} S_{*}^{n}\right) \neq 0$ for some $0<s$ and $s \neq n$.

Our approach will consist of relating the homotopic/homological properties of the embedding $S_{\infty}^{n} \hookrightarrow S_{\infty}^{n+2}$ to the corresponding properties for $S_{*}^{n} \hookrightarrow S_{*}^{n+2}$ (which we know to be non-trivially knotted). We first consider the case where knottedness is detected by the homology of the canonical infinite cyclic cover, by making the additional assumption:

Case 1: $H_{s}\left(\widehat{S_{*}^{n+2}-} S_{*}^{n}\right) \neq 0$ for some $s \neq 0, n$.

Now let $N_{1}, N_{2}$ be a pair of closed tubular neighborhoods of $S_{*}^{n} \subset S_{*}^{n+2}$ such that $N_{1} \subset \operatorname{Int}\left(N_{2}\right)$. Note that $N_{1}$ and $N_{2}$ are both homeomorphic to $S^{n} \times \mathbb{D}^{2}$ (since the embedding $S_{*}^{n} \subset S_{*}^{n+2}$ is tame). In fact, there is a homeomorphism taking $S_{*}^{n} \subset N_{1} \subset N_{2}$ to $S^{n} \times\{0\} \subset S^{n} \times \frac{1}{2} \mathbb{D}^{2} \subset S^{n} \times \mathbb{D}^{2}$ (where $\mathbb{D}^{2}$ refers to the unit disk in $\mathbb{R}^{2}$ ). Let $C=S_{*}^{n+2}-N_{1}$, and observe that $C$ is an open codimension zero submanifold of $S_{*}^{n+2}$ homeomorphic to the knot complement $S_{*}^{n+2}-S_{*}^{n}$. Furthermore, the inclusion $C \subset S_{*}^{n+2}-S_{*}^{n}$ is a homotopy equivalence.

Proposition 5. The homomorphism:

$$
i_{*}: H_{s}\left(\widehat{C_{\infty}}\right) \rightarrow H_{s}\left(\widehat{S_{\infty}^{n+2}-} S_{\infty}^{n}\right)
$$

which is induced by the inclusion map

$$
i: \widehat{C_{\infty}} \rightarrow S_{\infty}^{n+2}-S_{\infty}^{n}
$$

is non-zero.

Proof (Proposition 5). Express $S_{\infty}^{n+2}-S_{\infty}^{n}$ as the union of the following two open sets

$$
\begin{gathered}
A=C_{\infty} \\
B=\left(\operatorname{Int}\left(N_{2}\right)\right)_{\infty}-S_{\infty}^{n} .
\end{gathered}
$$

Note that $A \cap B=\left(\operatorname{Int}\left(N_{2}\right)-N_{1}\right)_{\infty}$. This implies that $\widehat{A} \cup \widehat{B}=\widehat{S_{\infty}^{n+2}-} S_{\infty}^{n}$ and $\widehat{A} \cap \widehat{B}=\widehat{A \cap B}$, so applying the Mayer-Vietoris sequence in dimension $s$, we get:

$$
H_{s}(\widehat{A} \cap \widehat{B}) \rightarrow H_{s}(\widehat{A}) \oplus H_{s}(\widehat{B}) \rightarrow H_{s}\left(S_{\infty}^{n+2}-S_{\infty}^{n}\right) .
$$

Hence to verify Proposition 5 , it suffices to show $(1) H_{s}(\widehat{A}) \neq 0$, and $(2) H_{s}(\widehat{A} \cap$ $\widehat{B})=0$. 
To see (1), apply Proposition $2^{\prime}$ to the set $U=C$. This yields an isomorphism $H_{s}(\widehat{A}) \cong H_{s}(\widehat{C})$. But since $C \subset S_{*}^{n+2}-S_{*}^{n}$ is a homotopy equivalence, and as we are working under the hypothesis of Case 1 , we have that $H_{s}(\widehat{C}) \neq 0$, establishing (1).

To see (2), apply Proposition $2^{\prime}$ to the set $U=\operatorname{Int}\left(N_{2}\right)-N_{1}$ (note that $\widehat{A} \cap \widehat{B}=$ $\left(\operatorname{Int}\left(\widehat{\left.N_{2}\right)-} N_{1}\right)_{\infty}\right)$. This yields an isomorphism $H_{s}(\widehat{A} \cap \widehat{B}) \cong H_{s}\left(\operatorname{Int} \widehat{\left(N_{2}\right)}-N_{1}\right)$. But $\operatorname{Int}\left(N_{2}\right)-N_{1}$ is homeomorphic to $S^{n} \times\left(S^{1} \times\left(\frac{1}{2}, 1\right)\right)$ by the discussion above. And hence $\operatorname{Int}\left(\widehat{\left.N_{2}\right)}-N_{1}\right.$ is homeomorphic to $S^{n} \times \mathbb{R}^{2}$. Since $s \neq 0, n$, we have that $H_{s}\left(S^{n} \times \mathbb{R}^{2}\right)=0$, establishing (2), and completing the proof of Proposition 5 .

Now Proposition 5 tells us that the embedding $S_{\infty}^{n} \hookrightarrow S_{\infty}^{n+2}$ is a knotted sphere, concluding the argument for Case 1 . We now focus on:

Case 2: $\pi_{1}\left(S_{*}^{n+2}-S_{*}^{n}\right) \neq \mathbb{Z}$.

In this case, we claim that $\pi_{1}\left(S_{\infty}^{n+2}-S_{\infty}^{n}\right)$ is likewise $\neq \mathbb{Z}$. In order to see this, consider the sets $N_{1}, N_{2}$, and $C$ defined previously. We have that $S_{*}^{n} \subset N_{1} \subset N_{2}$, with each $N_{i}$ a closed tubular neighborhood of $S_{*}^{n}$ in $S_{*}^{n+2}$, and $C$ is the set $S_{*}^{n+2}-N_{1}$.

Proposition 6. The image of the homomorphism:

$$
i_{\#}: \pi_{1}\left(C_{\infty}\right) \rightarrow \pi_{1}\left(S_{\infty}^{n+2}-S_{\infty}^{n}\right)
$$

which is induced by the inclusion map is a non-abelian group.

Proof (Proposition 6). As in the previous proposition, we decompose $S_{\infty}^{n+2}-S_{\infty}^{n}$ into a pair of open sets:

$$
\begin{gathered}
A=C_{\infty} \\
B=\left(\operatorname{Int}\left(N_{2}\right)\right)_{\infty}-S_{\infty}^{n}
\end{gathered}
$$

Note that $D:=A \cap B=\left(\operatorname{Int}\left(N_{2}\right)-N_{1}\right)_{\infty}$, and $A \cup B=S_{\infty}^{n+2}-S_{\infty}^{n}$. Applying Siefert-Van Kampen, we see that:

$$
\pi_{1}\left(S_{\infty}^{n+2}-S_{\infty}^{n}\right)=\pi_{1}(A) *_{1}(D) \pi_{1}(B)
$$

In order to show that $i_{\#}\left(\pi_{1}(A)\right)$ is non-abelian, it is sufficient to have $(1) \pi_{1}(A)$ is non-abelian, and (2) $i_{\#}$ is a monomorphism. 
Note that, applying Proposition 2 with $U=C$, we get that $\pi_{1}(A) \cong \pi_{1}(C)$. Since $C \subset S_{*}^{n+2}-S_{*}^{n}$ is a homotopy equivalence, and as we are working under the hypothesis of Case 2, we have that $\pi_{1}(A)$ is a non-abelian group (since it's abelianization is $H_{1}(A)=\mathbb{Z}$, while $\pi_{1}(A) \neq \mathbb{Z}$ ). This gives assertion (1).

Likewise, we can apply Proposition 2 with $U=\operatorname{Int}\left(N_{2}\right)-N_{1}$, obtaining an isomorphism $\pi_{1}(D) \cong \pi_{1}\left(\operatorname{Int}\left(N_{2}\right)-N_{1}\right)$. But $\operatorname{Int}\left(N_{2}\right)-N_{1}$ is homeomorphic to $S^{n} \times\left(S^{1} \times\left(\frac{1}{2}, 1\right)\right)$ by the discussion above, which implies that $\pi_{1}(D)=\mathbb{Z}$. Hence to get assertion (2), it is enough to show that $\pi_{1}(D)$ injects into $\pi_{1}\left(S_{\infty}^{n+2}-S_{\infty}^{n}\right)$. In order to see this, observe that we have the commutative diagram:

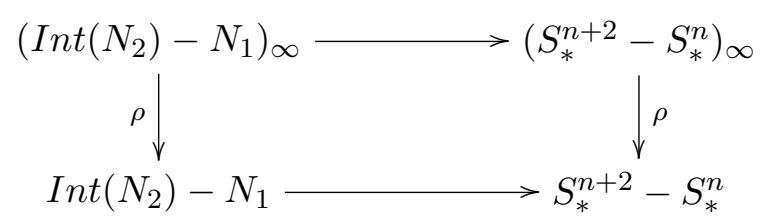

where the vertical arrows are given by geodesic retraction, and the horizontal arrows are inclusions. Applying the $H_{1}$ functor we obtain:

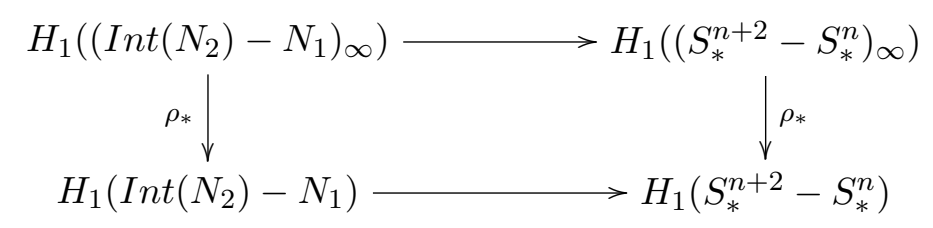

Proposition 2 tells us that the vertical maps are isomorphisms. Since the inclusion map $\operatorname{Int}\left(N_{2}\right)-N_{1} \subset S_{*}^{n+2}-S_{*}^{n}$ induces an isomorphism on $H_{1}$, the bottow arrow in the commutative diagram is also an isomorphism. This yields that the inclusion $D=\left(\operatorname{Int}\left(N_{2}\right)-N_{1}\right)_{\infty} \subset\left(S_{*}^{n+2}-S_{*}^{n}\right)_{\infty}$ induces an isomorphism on $H_{1}$. Finally, we note that we have a commutative diagram:

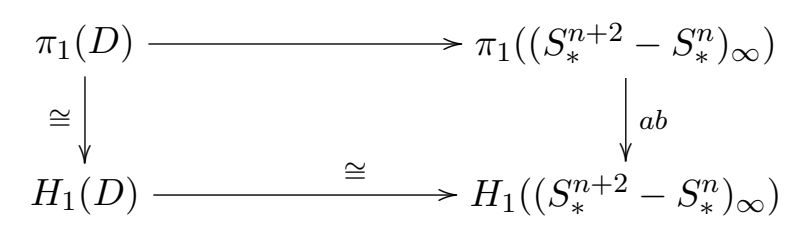

where the horizontal arrows are induced by the inclusion $D \subset\left(S_{*}^{n+2}-S_{*}^{n}\right)_{\infty}$, while the vertical arrows are given by abelianization. Since $\pi_{1}(D)=\mathbb{Z}$, abelianization gives an isomorphism. By the argument above, the inclusion $D \subset\left(S_{*}^{n+2}-S_{*}^{n}\right)_{\infty}$ induces an isomorphism on $H_{1}$. This implies that the inclusion $D \subset\left(S_{*}^{n+2}-S_{*}^{n}\right)_{\infty}$ 
induces a monomorphism on $\pi_{1}$, giving us assertion (2), and hence, completing the proof of the Proposition.

Proposition 6 now tells us that $\pi_{1}\left(S_{\infty}^{n+2}-S_{\infty}^{n}\right) \neq \mathbb{Z}$, and hence the embedding $S_{\infty}^{n} \hookrightarrow S_{\infty}^{n+2}$ is also knotted in Case 2. This completes the proof of the second case, and hence of Theorem 1.2.

Proof (Corollary 1.4). We start by observing that our hypotheses ensure the existence of a triangulation of the pair $\left(S^{n+2}, S^{n}\right)$ such that the involution $\tau$ is a simplicial map. Now recall that the strict hyperbolization procedure of CharneyDavis (section 7 in [6]) takes a simplicial complex and functorially assigns to it a topological space (in fact, a union of compact hyperbolic manifolds with corners) that supports a metric of strict negative curvature (i.e. a locally CAT(-1) metric). By functoriality we mean that simplicial isomorphisms induce isometries of the resulting spaces. Let us apply this procedure to the suspension of the sphere $\Sigma S^{n+2}$ (respectively $\Sigma S^{n}$ ), and call the resulting space $X$ (respectively $Y$ ).

Observe that the involution $\tau$ induces an involution on $\Sigma S^{n+2}$ with fixed point set $\Sigma S^{n}$, which by functoriality of the hyperbolization procedure, yields an isometric involution $\tau_{h}$ of $X$ with fixed point set the totally geodesic subspace $Y$. Now $\tilde{\tau}_{h}$ is a lift of this involution to the universal cover $\tilde{X}$, and the fixed set of $\tilde{\tau}_{h}$ will be a lift $\tilde{Y}$ of $Y$. It is easy to see that the fixed point set for the induced involution $\tau_{\infty}$ on the boundary at infinity is precisely the embedded $\partial^{\infty} \tilde{Y} \subset \partial^{\infty} \tilde{X}$. By our theorem, this is a knotted, totally wild, codimension two embedding of $S^{n}$ into $S^{n+2}$.

Proof (Corollary 1.5). We note that in the case where we start with a PLtriangulation of a smooth manifold representing its underlying PL-structure, the hyperbolization naturally carries a canonical smooth structure (see CharneyDavis [6]; smoothing theory [16] is also used). In particular, the space $X$ constructed in the previous Corollary is a well-defined smooth closed manifold, equipped with a locally CAT(-1) metric. Furthermore, the involution $\tau$ is a PL involution of $X$. In the notation of the statement of our Corollary, we are letting $M^{n+3}=X$ and $\sigma=\tau$.

We now claim that either $M^{n+3}$ supports no negatively curved Riemannian metric, or that $\sigma$ is not homotopic to an involutive isometry for any negatively curved Riemannian metric. To argue by contradiction, let us assume that 
there exists a negatively curved Riemannian metric $g$, an involutive isometry $\sigma^{\prime}:\left(M^{n+3}, g\right) \rightarrow\left(M^{n+3}, g\right)$, and a homotopy $\sigma^{\prime} \simeq \sigma$. Since $\sigma^{\prime}$ is homotopic to $\sigma$, we have that the induced maps $\sigma_{\infty}^{\prime}$ and $\sigma_{\infty}$ coincide on $\partial^{\infty} \tilde{M}^{n+3}$ (the lift $\tilde{\sigma}^{\prime}$ is the end of the lift of the homotopy that starts with the involution $\tilde{\sigma}$; it can then be shown that $\tilde{\sigma}^{\prime}$ is also an involution). In particular, the fixed point set of $\sigma_{\infty}^{\prime}$ is a knotted, totally wild embedding of $S_{\infty}^{n}$ in $\partial^{\infty} \tilde{M}^{n+3}=S_{\infty}^{n+2}$. Since $\sigma^{\prime}$ is an isometric involution on $\left(M^{n+3}, g\right)$, the fixed point set of $\sigma^{\prime}$ is a totally geodesic submanifold $N \subset M^{n+3}$. Since $\partial^{\infty} N$ must coincide with the fixed point set of the $\sigma_{\infty}^{\prime}$, the submanifold $N$ must have codimension two.

Now consider the isometric embedding $\tilde{N}^{n+1} \subset \tilde{M}^{n+3}$ which is left invariant under the lift $\tilde{\sigma}^{\prime}$ of $\sigma^{\prime}$, and let $* \in \tilde{N}^{n+1}$ be an arbitrary point. Let $K$ denote a sphere of radius $\epsilon$ centered at $*$. Since we are in a Riemannian manifold of negative curvature, geodesic retraction gives an actual homeomorphism from the knotted pair $\left(S_{\infty}^{n+2}, S_{\infty}^{n}\right)$ to the pair $\left(K, K \cap \tilde{N}^{n+1}\right)$. Finally, we note that $K$ is homeomorphic to $S^{n+2}$, and that $K \cap \tilde{N}^{n+1}$ is an unknotted $S^{n}$ in $K$ (as it is the intersection of a small metric sphere with a totally geodesic submanifold passing through the point $*$ ). This gives us a contradiction, and completes the proof of the Corollary.

\section{Appendix: Classification of tame knots in Dimension $\geq 6$.}

In this appendix, we provide a proof of the classification of tame knots in high dimensional spheres. While this result is probably known to experts, we were unable to find a reference in print.

Theorem 4.1. Let $S^{n} \hookrightarrow S^{n+2}$ be a tame knot, and let $W^{n+2}$ be the complement of a small open tubular neighborhood of $S^{n}$ in $S^{n+2}$ (note that $W^{n+2}$ is a compact manifold with boundary). Suppose that $n \geq 4$ (so the ambient sphere has dimension $\geq 6$ ) and that $\pi_{1}(W)=\mathbb{Z}$. Then there exists an integer $s$ satisfying:

- $0<s$ and $s \neq n$,

- $H_{s}(\tilde{W}) \neq 0$.

Before starting the proof, we observe that, if $\pi_{1}(W)=\mathbb{Z}$, then the covering $\widehat{W}$ of $W$ corresponding to the abelianization of $\pi_{1}$ coincides with the universal cover $\tilde{W}$ of $W$. 
Proof. Note first that if $\tilde{H}_{i}(\tilde{W})=0$ for all $i$, then $\tilde{W}$ is contractible, and hence $W$ is a homotopy circle. Then the fibering theorem of Browder-Levine [4], as extended to the topological category by the work of Kirby-Seibenmann [16], can be used to conclude that $S^{n} \hookrightarrow S^{n+2}$ is unknotted. This contradiction shows that we can assume that $H_{i}(\tilde{W}) \neq 0$ for some $0<i \leq n+2$. Now suppose that the only such integer $s$ such that $\tilde{H}_{s}(\tilde{W}) \neq 0$ is $s=n$. We will show this assumption leads to a contradiction, completing the proof of the theorem.

Observe that our assumption implies that $\pi_{i}(\tilde{W})=0$ for all $i<n$, and hence that $W$ is a compact manifold with the $n-1$ homotopy type of $S^{1}$ (in fact, a $K(\mathbb{Z}, 1)$ can be constructed by adding $n+1, n+2, \ldots$ cells to $W)$. Consequently, $H_{c}^{i}(\tilde{W})=H^{i}(\mathbb{Z}, \mathbb{Z}[\mathbb{Z}])$ for all $i \leq n-1$, and since $2<n-1$, we conclude that $H_{c}^{2}(\tilde{W})=0$ since the cohomological dimension of $\mathbb{Z}$ is one. But on the other hand, Lefschetz duality yields that $H_{n}(\tilde{W}) \cong H_{c}^{2}(\tilde{W}, \partial \tilde{W})$. Applying the long exact sequence for cohomology with compact supports to the pair $(\tilde{W}, \partial \tilde{W})$ yields:

$$
0=H_{c}^{2}(\tilde{W}) \leftarrow H_{n}(\tilde{W}) \leftarrow H_{c}^{1}(\partial \tilde{W}) \leftarrow H_{c}^{1}(\tilde{W})
$$

Since $\partial W=S^{1} \times S^{n}$, the same reasoning given above for $W$ yields that the inclusion $\partial W \rightarrow W$ is an $(n-1)$-equivalence, and hence the inclusion map $\partial \tilde{W} \rightarrow$ $\tilde{W}$ induces an isomorphism on the functor $H_{c}^{1}(-)$. This gives the contradiction $H_{n}(\tilde{W})=0$, completing the proof of the theorem.

Remark 6 . We point out that, in the case $n=1,2$, a tame knot $S^{n} \hookrightarrow S^{n+2}$ is non-trivial if and only if $\pi_{1}\left(S^{n+2}-S^{n}\right) \neq \mathbb{Z}$. For $n=1$, this is due to Papakyriakopoulos [19], while for $n=2$, this is Thm. 11.7.A in Freedman-Quinn [12]. In particular, there are algebraic criterions for deciding whether a tame embedding of a codimension two sphere is knotted when the ambient sphere has dimension $\neq 5$.

\section{Concluding Remarks.}

The smooth manifolds constructed in our Theorem 1.2 naturally come equipped with a locally $\mathrm{CAT}(-1)$ metric. It is reasonable to ask:

Question: Can these manifolds support Riemannian metrics of strictly negative curvature? 
Note that an answer in either direction would be of considerable interest. A negative answer would yield examples of locally CAT(-1) manifolds with a spherical boundary at infinity, but which nevertheless don't support any negatively curved Riemannian metric. This would further illustrate the gap between "metric" negative curvature and Riemannian negative curvature.

On the other hand, a positive answer would shed some cautionary light on the Nielson realization problem (the open problem mentioned in our Remark 4). Indeed, it would imply that for a closed negatively curved Riemannian manifold, the involutive homeomorphism $f: M \rightarrow M$ predicted by the Nielson realization problem might bear no obvious relationship to the geometry of any negatively curved Riemannian metric on $M$.

Next we observe that in the examples we constructed in Theorem 1.2, it was precisely the "local knottedness" of $Y$ in $X$ that allowed us to show that the embedding of the boundaries at infinity were knotted. One can ask whether this "local knottedness" is really necessary.

Question: Does there exist a compact, piecewise hyperbolic, locally CAT(-1) manifold $X$, containing a totally geodesic submanifold $Y$, whose universal covers have boundaries at infinity $S_{\infty}^{n+2}$ and $S_{\infty}^{n}$ respectively, and having the properties:

- at each point $y \in Y$, we have that $l k_{Y}(y) \hookrightarrow l k_{X}(y)$ is unknotted (i.e. $Y$ is a locally flat topological submanifold of $X$ ).

- $S_{\infty}^{n} \hookrightarrow S_{\infty}^{n+2}$ is knotted.

It would be quite interesting if the answer to this last question were positive. Finally, it is perhaps interesting to observe a formal analogy of Theorem 1.1 with a totally unrelated result in algebraic K-theory; i.e. the main result in Farrell [10]. That result asserts, for every ring $R$, that the abelian group $\operatorname{Nil}(R)$ is either zero, or not finitely generated.

\section{BiBLIOGRAPHY}

[1] Bass, H. \& Morgan, J.W. The Smith conjecture, Academic Press, Orlando, FL. 1984, xv+243 pp. 
[2] Bestvina, M. Local homology properties of boundaries of groups, Michigan Math. J. 43 (1996), pp. 123-139.

[3] Bing, R.H. The geometric topology of 3-manifolds, Amer. Math. Soc., Providence, RI. 1983, x+238 pp.

[4] Browder, M. \& Levine, J. Fibering manifolds over a circle, Comment. Math. Helv. 40 (1966), pp. 153-160.

[5] Brown, M. Some applications of an approximation theorem for inverse limits, Proc. Amer. Math. Soc. 11 (1960), pp. 478-483.

[6] Charney, R.M. \& Davis, M. W. Strict hyperbolization, Topology 34 (1995), no. 2 , pp. 329-350.

[7] Davis, M.W. \& Januszkiewicz, T. Hyperbolization of polyhedra, J. Differential Geom. 34 (1991), no. 2, pp. 347-388.

[8] Dydak, J. \& Segal, J. Shape theory: an introduction, Springer-Verlag, Berlin. 1978, vi+150 pp.

[9] Edwards, R.D. The topology of manifolds and cell-like maps, in Proceedings of the I.C.M., Helsinki (pp. 111-127). Acad. Sci. Fennica, Helsinki, 1980.

[10] Farrell, F.T. The non-finiteness of Nil, Proc. Amer. Math. Soc. 65 (1977), pp. 215-216.

[11] Farrell, F.T. \& Lafont, J.-F. Finite automorphisms of negatively curved Poincaré Duality groups, Geom. Funct. Anal. 14 (2004), pp. 283-294.

[12] Freedman, M.H. \& Quinn, F. Topology of 4-manifolds, Princeton University Press, Princeton, NJ. 1990, viii+259 pp.

[13] Giffen, C.H. The generalized Smith conjecture, Amer. J. Math. 88 (1966), pp. 187-198.

[14] Hirzebruch, F. Singularities and exotic spheres, Sém. Bourbaki Vol. 10 (1966/1967), No. 314., pp. 13-32, Soc. Math. France, Paris, 1995. 
[15] Kirby, R.C. \& Siebenmann, L.C. Normal bundles for codimension 2 locally flat imbeddings, in Geometric Topology (Proc. Conf., Park City, Utah, 1974) (pp. 310-324). Springer, Berlin, 1975.

[16] Kirby, R.C. \& Siebenmann, L.C. Foundational essays on topological manifolds, smoothings, and triangulations, Princeton University Press, Princeton, NJ. 1977, vii+355 pp.

[17] Lü, Z. A note on Brieskorn spheres and the generalized Smith conjecture, Michigan Math. J. 47 (2000), no. 2, pp. 325-333.

[18] Neumann, W.D. The fixed group of an automorphism of a word hyperbolic group is rational, Invent. Math. 110 (1992), no. 1, pp. 147-150.

[19] Papakyriakopoulos, C.D. On Dehn's Lemma and the asphericity of knots, Ann. Math. 66 (1957), pp. 1-26.

[20] Siebenmann, L.C. Approximating cellular maps by homeomorphisms, Topology 11 (1972), pp. 271-294.

F. T. Farrell

Department of Mathematical Sciences

Binghamton University

Binghamton, NY 13902-6000

E-mail: farrell@math.binghamton.edu

J.-F. Lafont

Department of Mathematics

The Ohio State University

Columbus, OH 43210

E-mail: jlafont@math.ohio-state.edu 